\title{
Contribution of UGT1A1 variations to chemotherapy-induced unconjugated hyperbilirubinemia in pediatric leukemia patients
}

\author{
Akitaka Nomura', Yoshihiro Maruo ${ }^{1}$, Takashi Taga ${ }^{1}$ and Yoshihiro Takeuchi ${ }^{1}$
}

BACKGROUND: Chemotherapy for malignant neoplasms sometimes induces unconjugated hyperbilirubinemia, resulting in the early cessation of treatment. We evaluated the role of variations in the bilirubin uridine-5-diphosphate (UDP)glucuronosyltransferase gene (UGT1A1) in unconjugated hyperbilirubinemia development during chemotherapy in pediatric patients with leukemia.

METHODS: UGT1A1 allelic variations were evaluated in 25 Japanese pediatric leukemia patients with hyperbilirubinemia (peak serum bilirubin concentration $3.57 \pm 1.02 \mathrm{mg} / \mathrm{dl}$ ) and 25 control patients without hyperbilirubinemia $(0.92 \pm 0.32 \mathrm{mg} / \mathrm{dl})$ by PCR-direct sequencing.

RESULTS: In the hyperbilirubinemic group, 22 of 25 patients showed biallelic variations of UGT1A1. Nine (36\%) patients were homozygous for UGT1A1*6 and eight (32\%) were compound heterozygous for UGT1A1*6 and UGT1A1*28. Three (12\%) patients were homozygous for UGT1A1*28. There were no biallelic variations in UGT1A1 in the non-hyperbilirubinemic group. The allelic frequencies of UGT1A1*6 in the hyperbilirubinemic group (0.58) was significantly higher than those of the non-hyperbilirubinemic group $(0.1)\left(\chi^{2}=25.7, P<0.05\right)$.

CONCLUSION: The high frequency of biallelic variations of UGTIA1 in the hyperbilirubinemic group suggests an association with Gilbert syndrome. Therefore, it is not necessary to cease chemotherapy in patients with these mutations who develop unconjugated hyperbilirubinemia without associated liver dysfunction.

C hemotherapy for malignant neoplasms sometime induces unconjugated hyperbilirubinemia without liver dysfunction, particularly in patients with leukemia. This hyperbilirubinemia is usually mild and transient, and is thought to result from chemotherapy-induced liver damage. In certain cases, according to the therapy protocol, chemotherapy must be suspended when such hyperbilirubinemia presents. Delayed chemotherapy poses a risk for patients in achieving or maintaining remission of the leukemia (1). We previously reported an association between chemotherapy-induced unconjugated hyperbilirubinemia and mutations of a bilirubin uridine-5-diphosphate (UDP)-glucuronosyltransferase gene: UDP-glucuronosyltransferase family 1 , polypeptide A1 (UGT1A1) (2). Bilirubin is selectively catalyzed by UGT1A1 (EC 2.4.1.17). Accordingly, mutations and polymorphisms of UGT1A1 cause hereditary forms of unconjugated hyperbilirubinemia, including Crigler-Najjar syndrome type I (MIM \#218800) and type II (MIM \#606785), and Gilbert syndrome (MIM \#143500). In particular, the mild hyperbilirubinemia occurring during chemotherapy has been associated with polymorphisms of UGT1A1 that cause Gilbert syndrome (3).

Gilbert syndrome is a form of mild hereditary unconjugated hyperbilirubinemia without liver dysfunction or hemolytic anemia (4), and is caused by biallelic variations of UGT1A1. Gilbert syndrome is one of the most prevalent congenital metabolic disorders with a prevalence of $3-8.6 \%$ of the population $(5,6)$. UGT1A1 consists of five exons, a TATA box, and a phenobarbital responsive enhancer module (gtPBREM) $(7,8)$. The wild type of the TATA box has six repeats of TA, and is conventionally written as A(TA)6TAA (c.-53TA[7]). The gtPBREM is an important enhancer module of UGT1A1, comprising a region of $290 \mathrm{bp}$ located approximately $3.5 \mathrm{~kb}$ upstream of the coding region, and works in the presence of nuclear receptors such as constitutive active receptor (8). There are two common polymorphic variations of UGT1A1: a TA-insertion mutation in the TATA box [A(TA)7TAA] with c. $-3279 \mathrm{~T}>\mathrm{G}$ in the gtPBREM [c.-3279T $>\mathrm{G} ; \mathrm{A}(\mathrm{TA}) 7 \mathrm{TAA}]$ $\left(U G T 1 A 1^{*} 28\right)$ and c.211G $>$ A (p.G71R) in exon 1 (UGT1A1*6) (5-8); A(TA)7TAA can also be indicated as c.- 53TA[8] according to the Human Genome Variation Society nomenclature. We revealed that A(TA)7TAA is usually linked with c. $-3279 \mathrm{~T}>\mathrm{G}$ in the gtPBREM [c.-3279T $>\mathrm{G} ; \mathrm{A}(\mathrm{TA}) 7 \mathrm{TAA}$ ], and this allele $\left(U G T 1 A 1^{*} 28\right)$ has been proposed to be essential in causing Gilbert syndrome (9). UGT1A $1^{\star} 28$ is found in all ethnic groups, and its frequency is $0.36-0.40$ in Caucasians, $0.35-$ 0.43 in Africans, and 0.15 in the Japanese population (10-12). This allele reduces the transcriptional activity of UGT1A1 to $30-69 \%$ of the wild-type enhancer-promoter $(10,13)$. Consequently, $U G T 1 A 1^{*} 28$ is considered to be the main cause of Gilbert syndrome. UGT1A1*6 (p.G71R) is another important polymorphism observed in the East Asian population

'Department of Pediatrics, Shiga University of Medical Science, Shiga, Japan. Correspondence: Akitaka Nomura (nomurara@belle.shiga-med.ac.jp) 
(14), which decreases UGT1A1 enzyme activity to $30-80 \%$ of the wild type $(15,16)$. The allelic frequency of $U G T 1 A 1^{\star} 6$ in East Asians is 0.16 (14), and has been reported as a risk factor of neonatal hyperbilirubinemia and a genetic cause of breast milk jaundice in this population $(14,17,18)$.

In patients with Gilbert syndrome, the jaundice usually becomes apparent during fasting, physical exercise, stress, infections, and menstruation (19). We previously published a case report of two patients with leukemia who were suffering from transient mild unconjugated hyperbilirubinemia during cancer chemotherapy without liver dysfunction (20). The aim of the present study was to investigate the contribution of UGT1A1 variants to chemotherapy-induced unconjugated hyperbilirubinemia in pediatric leukemic patients.

\section{RESULTS}

\section{Genotype Distributions in Patients With and Without Hyperbilirubinemia}

The genotype distributions of all patients are shown in Table 1. In the hyperbilirubinemia group, nine patients were homozygous for $U G T 1 A 1^{\star} 6(36 \%)$, eight patients were compound heterozygous for $U G T 1 A 1^{\star} 6$ and $U G T 1 A 1^{\star} 28(32 \%)$, and three patients were homozygous for UGT1A1*28 (12\%). One patient was compound heterozygous for $U G T 1 A 1^{\star} 6$ and $U G T 1 A 1^{\star} 7$ (c.1456T $>$ G, p.Y486D), and one was compound heterozygous for $U G T 1 A 1^{\star} 6$ and $U G T 1 A 1^{\star} 25$ (c.840C>A, p.C280X). Two patients were respectively heterozygous for $U G T 1 A 1^{\star} 6$ and $U G T 1 A 1^{\star} 28$. Overall, 22 of the 25 patients had biallelic variations $(88 \%)$. The allelic frequencies of $U G T 1 A 1^{\star} 6$ and $U G T 1 A 1^{\star} 28$ were 0.58 and 0.30 , respectively.

Genotype analysis of infants in the control group showed eight patients with the wild-type allele (homozygous $\left.U G T 1 A 1^{\star} 1\right)(32 \%)$, seven patients heterozygous for $U G T 1 A 1^{\star} 60$ (c. $\left.-3279 T>G\right)(28 \%)$, four patients heterozygous

Table 1. UGT1A1 genotype distributions in pediatric leukemia patients with and without hyperbilirubinemia

\begin{tabular}{|c|c|c|c|c|c|}
\hline \multirow{2}{*}{$\begin{array}{l}\text { Genotype } \\
\text { Allele } 1\end{array}$} & \multirow[b]{2}{*}{ Allele 2} & \multicolumn{2}{|c|}{$\begin{array}{l}\text { Hyperbilirubinemia } \\
\text { group }\end{array}$} & \multicolumn{2}{|c|}{$\begin{array}{l}\text { Non-hyperbilirubinemia } \\
\text { group }\end{array}$} \\
\hline & & $N=25$ & (\%) & $N=25$ & (\%) \\
\hline UGT1A ${ }^{*} 6$ & UGT1A1*6 & 9 & 36 & 0 & 0 \\
\hline UGT1A $1 * 6$ & $U G T 1 A 1 * 28$ & 8 & 32 & 0 & 0 \\
\hline UGT1A $1 * 28$ & UGT1A $1 * 28$ & 3 & 12 & 0 & 0 \\
\hline UGT1A1*6 & UGT1A $1 * 7$ & 1 & 4 & 0 & 0 \\
\hline UGT1A1*6 & UGT1A $1 * 25$ & 1 & 4 & 0 & 0 \\
\hline UGT1A1*6 & UGT1A $1 * 60$ & 0 & 0 & 2 & 8 \\
\hline UGT1A $1 * 60$ & UGT1A $1 * 60$ & 0 & 0 & 1 & 4 \\
\hline UGT1A1*6 & UGT1A $1 * 1$ & 1 & 4 & 3 & 12 \\
\hline UGT1A $1 * 28$ & $U G T 1 A 1 * 1$ & 1 & 4 & 4 & 16 \\
\hline UGT1A $1 * 60$ & UGT1A1*1 & 0 & 0 & 7 & 28 \\
\hline UGT1A1*1 & UGT1A1*1 & 1 & 4 & 8 & 32 \\
\hline
\end{tabular}

UGT1A1*1: wild-type allele; UGT1A*6:c.211G>A, p.G71R; UGT1A1*7:c.1456T>G, p.Y486D; UGT1A1*25: c.840C>A, p.C280X; UGT1A1*28: [c.-3279T>G; A(TA)7TAA]; UGT1A1*60: c.-3279T>G. for $U G T 1 A 1^{\star} 28(16 \%)$, three patients heterozygous for $U G T 1 A 1^{\star} 6(12 \%)$, two patients compound heterozygous for $U G T 1 A 1^{\star} 6$ and $U G T 1 A 1^{*} 60(8 \%)$, and one patient homozygous for $U G T 1 A 1^{\star} 60$ (4\%). None of these patients showed homozygous or compound heterozygous UGT1A1*6 and $U G T 1 A 1^{\star} 28$ genotypes. The allelic frequencies of $U G T 1 A 1^{\star} 6$ and $U G T 1 A 1^{*} 28$ in the control group were 0.1 and 0.08 , respectively. The allelic frequencies of $U G T 1 A 1^{\star} 6$ in the hyperbilirubinemic group (0.58) was significantly higher $\left(\chi^{2}=25.7\right.$; $P<0.05)$ than those of the non-hyperbilirubinemic group. The allelic frequencies of $U G T 1 A 1^{\star} 28$ was higher than the nonhyperbilirubinemic group, however is not significantly different $\left(\chi^{2}=7.86 ; P=0.05\right)$.

Characteristics of Patients With and Without Hyperbilirubinemia As shown in Table 2, in the hyperbilirubinemia group, the patients' peak serum bilirubin concentration ranged from 2.30 to $6.30 \mathrm{mg} / \mathrm{dl}$ (mean $3.57 \pm 1.02 \mathrm{mg} / \mathrm{dl}$ ). Three patients showed a Common Terminology Criteria for Adverse Events (CTCAE) grade of 2, and 22 patients showed a CTCAE grade of 3 . The peak serum indirect bilirubin level ranged from 1.50 to $5.50 \mathrm{mg} / \mathrm{dl}$ (mean $2.78 \pm 1.04 \mathrm{mg} / \mathrm{dl}$ ). The liver transaminase values were as follows: aspartate aminotransferase (AST) and alanine aminotransferase (ALT) ranged from 8 to $49 \mathrm{IU} / \mathrm{l}$ (mean $24.7 \pm 10.2 \mathrm{IU} / \mathrm{l}$ ) and from 9 to $88 \mathrm{IU} / 1$ (mean $35.7 \pm 20.8 \mathrm{IU} / \mathrm{l})$, respectively. In the control group, the peak serum bilirubin concentration ranged from 0.40 to $1.52 \mathrm{mg} /$ $\mathrm{dl}$ (mean $0.92 \pm 0.32 \mathrm{mg} / \mathrm{dl}$ ). The CTCAE grade was 0,1 , and 2 in 12,12 , and 1 patient, respectively. The peak serum indirect bilirubin level ranged from 0.09 to 1.20 (mean $0.77 \pm 0.26 \mathrm{mg}$ / dl). Liver transaminase values (AST and ALT) ranged from 17 to $48 \mathrm{IU} / \mathrm{l}(27.6 \pm 6.83 \mathrm{IU} / \mathrm{l})$ and from 10 to $53 \mathrm{IU} / \mathrm{l}(28.2 \pm 12.5$ IU/l), respectively. The $t$-test analysis showed a significant difference in the serum total bilirubin concentration between the two groups $(P<0.05$; Figure 1$)$. However, there was no significant difference in the transaminase (AST and ALT) levels between the two groups ( $P=0.111$ and 0.154 ; Figures 2 and 3 ).

\section{DISCUSSION}

Chemotherapy-induced unconjugated hyperbilirubinemia is recognized as an adverse event (AE) caused by anti-neoplasm agents, which results in suspension of the chemotherapy. We have observed some patients with chemotherapy-induced hyperbilirubinemia but without liver dysfunction. Our preliminary report demonstrated an association between such patients and UGT1A1 genotypes, indicating that UGT1A1 polymorphism is a risk for elevation of serum bilirubin concentration during cancer chemotherapy (20). We propose that if this hyperbilirubinemia is caused by the UGT1A1 variant, then it is not necessary to suspend the chemotherapy for convalescence. In the present study, we found that most of the patients in the hyperbilirubinemic group (22 of 25) harbored biallelic variants of UGT1A1. The most dominant genotype (observed in nine patients) was homozygous $U G T 1 A 1^{\star} 6$, which results from a transition of nucleotide c.211G $>$ A, leading to the replacement of glycine to arginine at codon 71 (p.G71R) and 


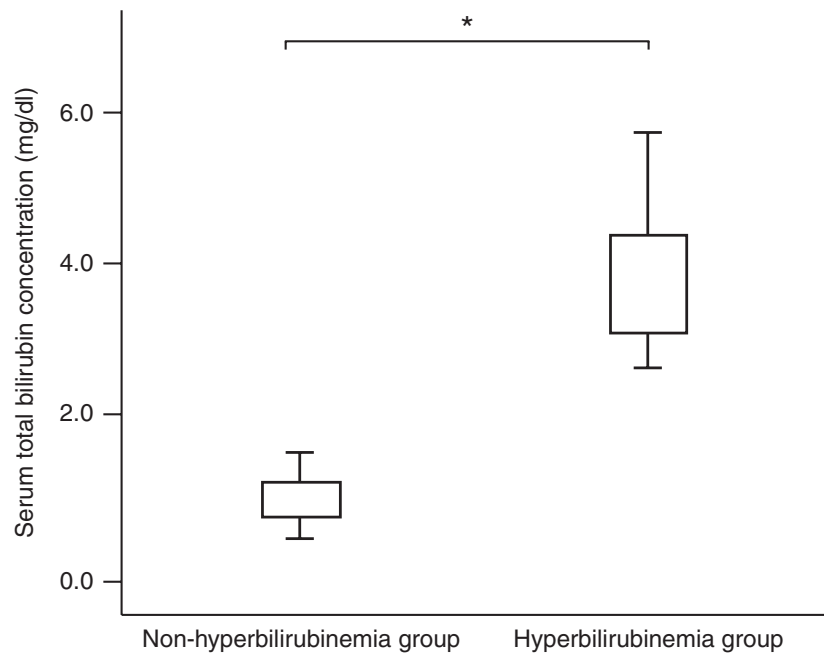

Figure 1. Comparison of serum total bilirubin between the control group and hyperbilirubinemia group. In the hyperbilirubinemia group, the patients' peak serum bilirubin concentration ranged from 2.30 to $6.30 \mathrm{mg} /$ $\mathrm{dl}$ (mean $3.57 \pm 1.02 \mathrm{mg} / \mathrm{dl}$ ). In the control group, the peak serum bilirubin concentration ranged from 0.40 to $1.52 \mathrm{mg} / \mathrm{dl}$ (mean $0.92 \pm 0.32 \mathrm{mg} / \mathrm{dl}$ ). The $t$-test showed a significant difference in serum total bilirubin concentration between the two groups $\left({ }^{*} P<0.05\right)$.

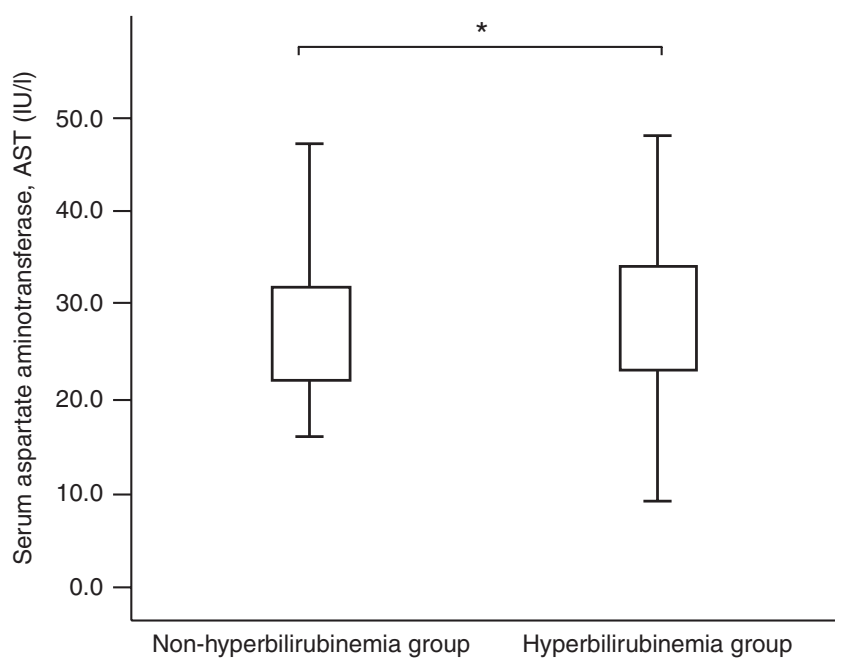

Figure 2. Comparison of serum aspartate aminotransferase levels in the control group and hyperbilirubinemia group. There was no significant difference between the two groups $\left({ }^{*} P=0.111\right)$.

reduced glucuronidation activity toward bilirubin. UGT1A1*6 is an important polymorphism as a cause of Gilbert syndrome and breast milk jaundice, and is also a risk factor for neonatal hyperbilirubinemia in the early neonatal period $(14,17)$. The allelic frequency of $U G T 1 A 1^{*} 6$ is 0.16 in the Japanese population. The other prevalent genotype (observed in eight patients) was compound heterozygous for $U G T 1 A 1^{*} 6$ and $U G T 1 A 1^{*} 28$. $U G T 1 A 1^{\star} 28$ is another important polymorphism that causes Gilbert syndrome, and is broadly observed worldwide $(10,11)$. The allelic frequency of $U G T 1 A 1^{\star} 28$ in the Japanese population is 0.15 . Three patients were homozygous for UGT1A1*28, which reduces the transcriptional activity of UGT1A1 by $30-$ $80 \%$ of the wild-type promoter-enhancer. The remaining two 


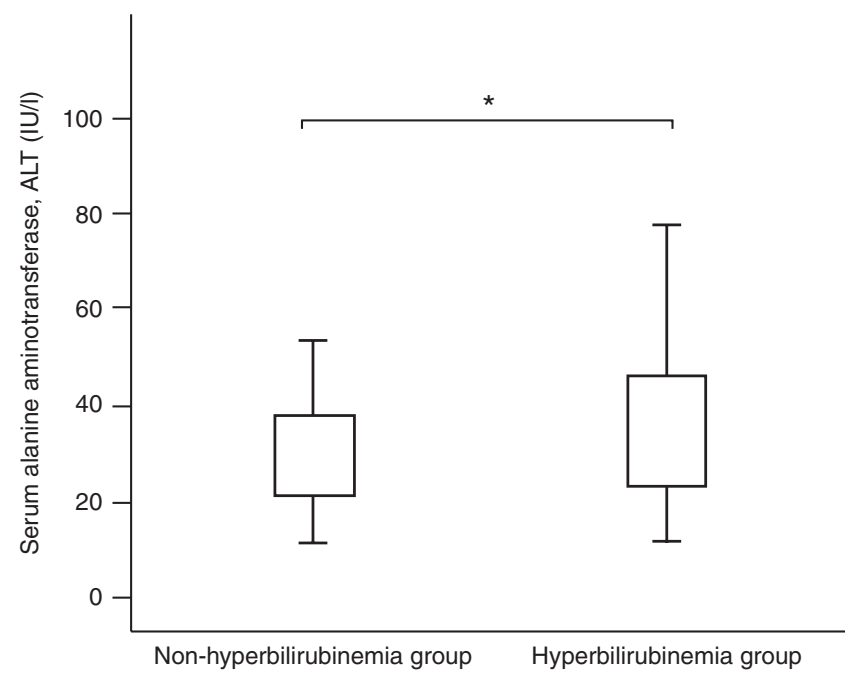

Figure 3. Comparison of serum alanine aminotransferase levels in the control group and hyperbilirubinemia group. There was no significant difference between the two groups ( $\left.{ }^{*} P=0.154\right)$.

patients were compound heterozygous for the disease-causing variants $U G T 1 A 1^{\star} 6 / U G T 1 A 1^{\star} 7$ and $U G T 1 A 1^{\star} 6 / U G T 1 A 1^{\star} 25$, respectively. $U G T 1 A 1^{\star} 25$ encodes p.C280X, and the homozygous form of p.C280X is the endemic mutation causing Crigler-Najjar syndrome type 1 in the Japanese population (21). Therefore, these results indicate that most of the cases of hyperbilirubinemia in this sample were cases of Gilbert syndrome. None of the patients in the non-hyperbilirubinemic group showed biallelic disease-causing variants of UGT1A1. One patient was compound heterozygous for $U G T 1 A 1^{\star} 6$ and $U G T 1 A 1^{\star} 60$ and two patients were homozygous for $U G T 1 A 1^{*} 60$, a polymorphism in gtPBREM (c. $-3279 \mathrm{~T}>\mathrm{G}$ ). The allelic frequencies of $U G T 1 A 1^{\star} 60$ are very high in every population examined to date: 0.26 in the Japanese population, 0.47 in Caucasians, and 0.85 in African-Americans (3). This polymorphism is not considered to be a cause of Gilbert syndrome and might be a modifier of the serum bilirubin level (22). Linkage of c.-3279T $>\mathrm{G}$ in gtPBREM and A(TA)7TAA in the TATA box $\left(U G T 1 A 1^{\star} 28\right)$ is essential to generate Gilbert syndrome $(9,22)$. None of the patients in the non-hyperbilirubinemic group had Gilbert syndrome.

These results suggest that chemotherapy-induced unconjugated hyperbilirubinemia without liver dysfunction is associated with Gilbert syndrome. Therefore, patients with biallelic UGT1A1 variants that are treated with chemotherapeutic agents might experience unconjugated hyperbilirubinemia as a symptom of Gilbert syndrome. The mechanism of the development of unconjugated hyperbilirubinemia is as follows. During cancer chemotherapy, chemotherapeutic agents usually cause emesis and loss of appetite, and patients enter a low caloric state. Fasting is known as one of the most important factors to induce hyperbilirubinemia in patients with Gilbert syndrome (23). Chemotherapeutic agents (such as 6-mercaptopurine, vincristine, mitoxantrone hydrochloride, and pirarubicin hydrochloride) may also induce hyperbilirubinemia, as do agents that inhibit DNA and RNA synthesis (24-26) and thus reduce the amount of bilirubin UDP-glucuronoyltransferase synthesized in hepatocytes. Patients with biallelic UGT1A1 variations produce a less efficient form of the enzyme, and they may show a transient elevation of the serum unconjugated bilirubin level. The simultaneous occurrence of these two factors during cancer chemotherapy might induce transient unconjugated hyperbilirubinemia in patients with Gilbert syndrome.

In the hyperbilirubinemic group, the CTCAE grade was significantly higher than that in the non-hyperbilirubinemic group. According to the Japanese Pediatric Leukemia/ Lymphoma Study Group protocol, cancer chemotherapy should be cancelled or delayed in the hyperbilirubinemic group to avoid lethal AEs. However, delayed chemotherapy increases the risk for relapse and insufficient induction of leukemia (1).

On the other hand, if leukemic patients with biallelic variation of UGT1A1 develop hyperbilirubinemia during cancer chemotherapy without liver dysfunction, this hyperbilirubinemia is not due to any $\mathrm{AE}$ associated with the chemotherapeutic agents but is rather due to Gilbert syndrome. Therefore, therapy should not be cancelled or suspended, and an appropriate therapy schedule can be maintained. Recently, Berrueco et al. (27) revealed that pediatric acute lymphoblastic leukemia (ALL) patients homozygous for the $U G T 1 A 1^{\star} 28$ allele tended to develop transient hyperbilirubinemia during cancer chemotherapy. They concluded that Gilbert syndrome should be taken into account when bilirubin levels increase without signs of hepatitis or hemolysis. An early diagnosis would avoid unnecessary changes in chemotherapy treatment. In our study, we did not evaluate thyroid function, haptoglobin levels, or cold agglutinin levels, and did not perform the direct antiglobulin test. Therefore, we could not completely rule out the involvement of hypothyroidism and autoimmune hemolytic anemia. However the result of our study supports their conclusion and $U G T 1 A 1^{\star} 6$, which is another important variant in East Asia, also likely plays a role in the development of chemotherapyinduced hyperbilirubinemia.

Gilbert syndrome is one of the most prevalent congenital metabolic disorders. In leukemic patients, the prevalence of Gilbert syndrome is estimated at $3-8.6 \%$ of the population. Therefore, before evaluation of $\mathrm{AE}$ due to anti-cancer agents using the CTCAE system, it is necessary to conduct a genetic analysis of UGT1A1 for patients with chemotherapy-induced hyperbilirubinemia.

\section{Conclusion}

Chemotherapy-induced unconjugated hyperbilirubinemia is a symptom of Gilbert syndrome. It is not necessary to cancel chemotherapy if biallelic UGT1A1 variants are confirmed in patients that develop unconjugated hyperbilirubinemia without liver dysfunction, since the symptom is due to Gilbert syndrome and does not indicate an $\mathrm{AE}$ of the chemotherapeutic agent.

\section{METHODS}

\section{Patients}

The characteristics of included patients are shown in Table 2. A total of 25 pediatric patients ( 13 males and 12 females, aged 3-16 y, median 
7.52 y) with acute leukemia (24 with ALL and 1 with acute myeloid leukemia) who showed transient unconjugated hyperbilirubinemia $(>2.0 \mathrm{mg} / \mathrm{dl})$ during cancer chemotherapy without liver dysfunction and hemolytic anemia were included in the study.

There were no patient that suspected hypothyroidism by the clinical condition and the family history.

After chemotherapy was complete, the serum bilirubin concentrations of these patients improved by less than $1 \mathrm{mg} / \mathrm{dl}$. The control group comprised 25 patients (aged 1-17 y; median, $5.84 \mathrm{y}$ ) with leukemia (20 with ALL and 5 with acute myeloid leukemia) who did not show unconjugated hyperbilirubinemia during cancer chemotherapy. The peak serum total bilirubin concentration was less than $2.0 \mathrm{mg} / \mathrm{dl}$ in the control group. This study was approved by the ethics committee of Shiga University of Medical Science.

\section{Evaluation of AEs}

AEs of the patients, including hyperbilirubinemia and transaminase levels, were evaluated using the CTCAE system (version 4.0), which is maintained by the US National Cancer Institute (28), and is widely used in oncology clinical practice to document the adverse effects of cancer treatment. The CTCAE grade reflects the severity of an AE on a scale of 1 to 5 with unique clinical descriptions based on this general guideline (where Grade 1 is mild and Grade 5 is lethal). The grading of hyperbilirubinemia is determined by the total serum bilirubin level, according to the upper limit of normal (ULN) value standardized for age (29).

Grade 1 (mild): <1.5× ULN; Grade 2 (moderate): >1.5-3.0× ULN; Grade 3 (severe): $>3.0-10.0 \times$ ULN; Grade 4 (disabling or life threatening): $>10.0 \times$ ULN; Grade 5: death. Grading of liver damage is determined by the levels of liver transaminases (AST and ALT) as follows: Grade 1 (mild): ULN-3.0× ULN; Grade 2 (moderate): >3.0$5.0 \times$ ULN; Grade 3 (severe): $>5.0-20.0 \times$ ULN; Grade 4 (disabling or life threatening): $>20.0 \times$ ULN; Grade 5: death. According to current guidelines, if pediatric leukemia patients develop more than Grade-3 hyperbilirubinemia during chemotherapy, even if without liver dysfunction and hemolytic anemia, it is necessary to cancel or delay the chemotherapy, or reduce the intensity.

\section{Sequence Analysis of UGT1A1}

For sequence analysis of $U G T 1 A 1$ variations, genomic DNA was isolated from the leukocytes of patients, after obtaining informed consent from the patients and parents. We amplified the exons, promoter region, and gtPBREM of UGT1A 1 from genomic DNA using PCR (18). In brief, approximately $100 \mathrm{ng}$ of total genomic DNA was amplified with pairs of oligonucleotide primers. Exons 2, 3, and 4 and their intervening introns were simultaneously amplified as a single DNA fragment using the primer pair $5^{\prime}$-CTCTATCTCAAACACGCATGCC-3'/ $5^{\prime}$-TTTTATCATGAATGCCATGACC-3'. The 50 region of UGT1A1, including the TATA box to exon 1, exon 5, and gtPBREM, was amplified separately with the primer pairs 5'-AAGTGAACTCCCTGCTACCTT$3^{\prime} / 5^{\prime}$-GCTTGCTCAGCATATATCTGGG-3' (the promoter region to exon 1); $5^{\prime}$-GAGGATTGTTCATACCACAGG-3'/5'-GCACTCTGG GGCTGATTAAT-3' (exon 5); and $5^{\prime}$-CTGGGGATAAACATGGG ATG-3'/5'-CACCACCACTTCTGGAACCT-3' (gtPBREM), respectively. The conditions for PCR were as follows: initial denaturation for $2 \mathrm{~min}$ at $94^{\circ} \mathrm{C}$, followed by $1 \mathrm{~min}$ at $94^{\circ} \mathrm{C}, 1 \mathrm{~min}$ at $60^{\circ} \mathrm{C}$, and $2 \mathrm{~min}$ at $72{ }^{\circ} \mathrm{C}$ for 30 cycles with a Minicycler (MJ Research, Watertown, MA). A final extension for $10 \mathrm{~min}$ at $72{ }^{\circ} \mathrm{C}$ was performed to ensure complete extension of PCR products. The sequences of the amplified DNA fragments were determined directly using the following sequencing primers: gtPBREM, 5'-TGAGTTTATATAACCTC-3'; TATA box and exon 1, 5' -CTATTTCATGTCCCCTCTGC-3', 5'-GT CTTTTGTTAGTCTCGGGC-3'， 5'-TTGTTGTGCAGTAAGTGGG A-3', $5^{\prime}$-CCATTCTCCTACGTGCCCAG-3' $3^{\prime}$ and $5^{\prime}$-AAGGGTTGC ATACGGGGAATA-3'; exon 2, 5'-GGAAGCTGGAAGTCTGGG-3'; exon 3, 5'-CTAGTTAGTATAGCAGAT-3'; exon 4: 5' -CAGCTGTG AAACTCAGAG-3; exon 5, 5'-TGCTGACAGTGGCCTTCATC-3', and $5^{\prime}$-GG TAGCCATAAGCACAACAT- ${ }^{\prime}$. The sequences of the amplified DNA fragments were determined directly using a BigDye Terminator v1.1 Cycle Sequencing Kit and Genetic Analyzer ABI Prism 3130xl (Applied Biosystems, Carlsbad, CA).

\section{Statistical Analysis}

The peak serum bilirubin concentration, and transaminase levels were statistically compared between the hyperbilirubinemic group and the control group using the $t$-test; $\chi^{2}$ analysis was conducted on the raw frequencies with IBM SPSS (version 22.0; IBM Corporation, Armonk, NY) and JMP9 (SAS Institute, Cary, NC).

\section{STATEMENT OF FINANCIAL SUPPORT}

This work was partly supported by a Grant-in-Aid for Scientific Research from the Ministry of Education, Science and Culture of Japan (grant number 15K09710).

Disclosure: The authors have nothing to disclose.

\section{REFERENCES}

1. Kishi S, Cheng C, French D, et al. Ancestry and pharmacogenetics of antileukemic drug toxicity. Blood 2007;109:4151-7.

2. Bosma PJ, Seppen J, Goldhoorn B, et al. Bilirubin UDP-glucuronosyltransferase 1 is the only relevant bilirubin glucuronidating isoform in man. J Biol Chem 1994;269:17960-4.

3. Maruo Y, Takahashi H, Matsui K, Sato H, Takeuchi Y. Phase II drug metabolism and individualized drug therapy: a focus on functional genetic variation in UDP-glucuronosyltransferases. Curr Pharmacogenomics Person Med 2010; 8:146-166.

4. Gilbert A, Lereboullet P. La cholémie simple familiale. Semaine Med 1901;21:241-3.

5. Owens D, Evans J. Population studies on Gilbert's syndrome. J Med Genet 1975;12:152-6.

6. Sieg A, Arab L, Schlierf G, Stiehl A, Kommerell B. [Prevalence of Gilbert's syndrome in Germany]. Dtsch Med Wochenschr 1987;112:1206-8.

7. Ritter JK, Chen F, Sheen YY, et al. A novel complex locus UGT1 encodes human bilirubin, phenol, and other UDP-glucuronosyltransferase isozymes with identical carboxyl termini. J Biol Chem 1992;267:3257-61.

8. Sugatani J, Kojima $\mathrm{H}$, Ueda A, et al. The phenobarbital response enhancer module in the human bilirubin UDP-glucuronosyltransferase UGT1A1 gene and regulation by the nuclear receptor CAR. Hepatology 2001;33:1232-8.

9. Maruo Y, D'Addario C, Mori A, et al. Two linked polymorphic mutations (A(TA)7TAA and T-3279G) of UGT1A1 as the principal cause of Gilbert syndrome. Hum Genet 2004;115:525-6.

10. Bosma PJ, Chowdhury JR, Bakker C, et al. The genetic basis of the reduced expression of bilirubin UDP-glucuronosyltransferase 1 in Gilbert's syndrome. N Engl J Med 1995;333:1171-5.

11. Monaghan G, Ryan M, Seddon R, Hume R, Burchell B. Genetic variation in bilirubin UPD-glucuronosyltransferase gene promoter and Gilbert's syndrome. Lancet 1996;347:578-81.

12. Sato $\mathrm{H}$, Adachi $\mathrm{Y}$, Koiwai $\mathrm{O}$. The genetic basis of Gilbert's syndrome. Lancet 1996;347:557-8.

13. Beutler E, Gelbart T, Demina A. Racial variability in the UDP-glucuronosyltransferase 1 (UGT1A1) promoter: a balanced polymorphism for regulation of bilirubin metabolism? Proc Natl Acad Sci USA 1998;95:8170-4.

14. Maruo Y, Nishizawa K, Sato H, Doida Y, Shimada M. Association of neonatal hyperbilirubinemia with bilirubin UDP-glucuronosyltransferase polymorphism. Pediatrics 1999;103(6 Pt 1):1224-7.

15. Yamamoto K, Sato H, Fujiyama Y, Doida Y, Bamba T. Contribution of two missense mutations (G71R and Y486D) of the bilirubin UDP glycosyltransferase (UGT1A1) gene to phenotypes of Gilbert's syndrome and Crigler-Najjar syndrome type II. Biochim Biophys Acta 1998;1406:267-73.

16. Ota Y, Maruo Y, Matsui K, Mimura Y, Sato H, Takeuchi Y. Inhibitory effect of $5 \beta$-pregnane-3 $\alpha, 20 \beta$-diol on transcriptional activity and enzyme activity of human bilirubin UDP-glucuronosyltransferase. Pediatr Res 2011;70:453-7.

17. Maruo Y, Nishizawa K, Sato H, Sawa H, Shimada M. Prolonged unconjugated hyperbilirubinemia associated with breast milk and mutations of the bilirubin uridine diphosphate- glucuronosyltransferase gene. Pediatrics 2000;106:E59.

18. Maruo Y, Morioka Y, Fujito H, et al. Bilirubin uridine diphosphate-glucuronosyltransferase variation is a genetic basis of breast milk jaundice. J Pediatr 2014;165:36-41.e1. 
19. Jansen PL, Bosma PJ, Chowdhury JR. Molecular biology of bilirubin metabolism. Prog Liver Dis 1995;13:125-50.

20. Maruo Y, Sato H, Bamba N, et al. Chemotherapy-induced unconjugated hyperbilirubinemia caused by a mutation of the bilirubin uridine5 '-diphosphate-glucuronosyltransferase gene. J Pediatr Hematol Oncol 2001;23:45-7.

21. Maruo Y, Ozgenc F, Mimura Y, et al. Compound heterozygote of a novel missense mutation (p.K402T) and a double missense mutation (p.[G71R;Y486D]) in type II Crigler-Najjar syndrome. J Pediatr Gastroenterol Nutr 2011;52:362-5.

22. Matsui K, Maruo Y, Sato H, Takeuchi Y. Combined effect of regulatory polymorphisms on transcription of UGT1A1 as a cause of Gilbert syndrome. BMC Gastroenterol 2010;10:57.

23. Ishihara T, Kaito M, Takeuchi K, et al. Role of UGT1A1 mutation in fasting hyperbilirubinemia. J Gastroenterol Hepatol 2001;16:678-82.

24. Wagner EK, Roizman B. Effect of the Vinca alkaloids on RNA synthesis in human cells in vitro. Science 1968;162:569-70.
25. Alberts DS, Peng YM, Bowden GT, Dalton WS, Mackel C. Pharmacology of mitoxantrone: mode of action and pharmacokinetics. Invest New Drugs 1985;3:101-7.

26. Kawahata RT, Chuang LF, Holmberg CA, Osburn BI, Chuang RY. Inhibition of human lymphoma DNA-dependent RNA polymerase activity by 6-mercaptopurine ribonucleoside triphosphate. Cancer Res 1983;43: 3655-9.

27. Berrueco R, Alonso-Saladrigues A, Martorell-Sampol L, et al. Outcome and toxicities associated to chemotherapy in children with acute lymphoblastic leukemia and Gilbert syndrome. Usefulness of UGT1A1 mutational screening. Pediatr Blood Cancer 2015;62:1195-201.

28. Basch E, Reeve BB, Mitchell SA, et al. Development of the National Cancer Institute's patient-reported outcomes version of the common terminology criteria for adverse events (PRO-CTCAE). J Natl Cancer Inst 2014;106:.

29. Common Terminology Criteria for Adverse Events (CTCAE) Version 4.0. http://evs.nci.nih.gov/ftp1/CTCAE/CTCAE_4.03. pdf. Accessed 28 May 2009. 